\title{
INFLUÊNCIA DA POSIÇÃO DO DUTO DE ENTRADA NO CICLONE COMO SECADOR: MODELAGEM E SIMULAÇÃO
}

\author{
J. A. R. SOUZA ${ }^{1}$, E. S. BARBOSA ${ }^{2}$, J. S. SOUZA ${ }^{3}$, S. R. FARIAS NETO ${ }^{4}$, A.G.B. LIMA ${ }^{3}$, F. P. M \\ FARIAS $^{5}$, H. M. LOPES \\ ${ }^{1}$ Universidade Federal de Campina Grande, Departamento de Física \\ ${ }^{2}$ Universidade Federal de Campina Grande, Departamento de Engenharia de Petróleo \\ ${ }^{3}$ Universidade Federal de Campina Grande, Departamento de Engenharia Mecânica \\ ${ }^{4}$ Universidade Federal de Campina Grande, Departamento de Engenharia Química \\ ${ }^{5}$ Universidade Federal de Campina Grande, Departamento de Tecnologia e Desenvolvimento \\ ${ }^{6}$ Universidade Federal de Campina Grande, Departamento de Engenharia Química
}

\section{E-mail para contato: jossyl@df.ufcg.edu.br}

\begin{abstract}
RESUMO - Secagem é a remoção, voluntária ou não, total ou parcial, de uma fase líquida ou gasosa de qualquer material, envolvendo a transferência de calor e massa. Nos últimos anos o ciclone vem sendo utilizado como uma alternativa de secagem de produtos agroindustriais. Neste sentido, propõe-se estudar numericamente a secagem do bagaço de cana-de-açúcar usando o ciclone como secador. Adotou-se a abordagem EulerianoLagrangeano em regime permanente com a partícula sendo considerada como uma mistura binária (bagaço de cana-de-açúcar e água). Adotou-se o modelo de turbulência das tensões de Reynolds (LRR-Launder-Reece-Rodi) para a fase gás, e um modelo de zero equação para a fase particulada, assumindo o modelo concentrado transiente de transferência de calor e massa para a fase particulada. As equações governantes foram resolvidas numericamente com auxílio de uma malha estruturada, utilizando elementos hexaédricos, representativa do ciclone secador com uma entrada tangencial proporcionando um escoamento no sentido anti-horário. Todas as simulações foram realizadas no Ansys CFX 12. Os resultados do teor de umidade, temperatura, trajetória das partículas, campos de velocidade, pressão e temperatura no interior do ciclone, são apresentados e discutidos.
\end{abstract}

\section{INTRODUÇÃO}

Tem sido reportado na literatura vários trabalhos relacionados à secagem de produtos biológicos utilizando-se diversos tipos de secadores, a exemplo de Mariani et al. (2008), Silva et al. (2009). O ciclone tem sido utilizado como secador, podendo-se destacar a secagem de produtos biológicos, (bagaço de cana-de-açúcar, borra de café, resíduos de laranja ou batatas) estudados por Corrêa (2003), Arnao et al. (2004), Corrêa (2004), Farias (2006). Os ciclones apresentam um comportamento fluidodinâmico complexo, apresentando, ao mesmo tempo, zonas de recirculação, reversão de fluxo, alta preservação de vórtices, grande perda de carga, entre outras. Porém, apesar de sua crescente 
utilização na indústria de secagem, apresentam algumas desvantagens: baixa eficiência para partículas com dimensões menores que $5 \mu \mathrm{m}$ e alta queda de pressão.

Daí a necessidade de se estudar e avaliar este dispositivo, visando reduzir custos operacionais e melhorar o seu desempenho, oferecendo, assim, mais uma alternativa como equipamento de secagem de produto biológico como, por exemplo, o bagaço de cana-de-açúcar. Além desse aspecto, deve-se considerar o fato de que a secagem de produtos biológicos envolve sistemas multifásicos e/ou multicomponentes e processos de transferência de massa e de calor e cinética de secagem, o que dificulta ainda mais o estudo do comportamento em ciclones. Poucos são os trabalhos que se propuseram a avaliar o ciclone como equipamento de secagem. Corrêa (2003) estudou teoricamente e experimentalmente a secagem do bagaço de cana-de-açúcar empregando dois equipamentos: uma câmara ciclônica e outro ciclone como secador. Mais tarde, Farias (2006) aprofundou os estudos do ciclone secador usado por Corrêa (2003) avaliando numericamente a influência da forma do duto de alimentação e do diâmetro da saída inferior do ciclone. Farias (2006) adotou o modelo EulerianoLagrangeano para o escoamento bifásico gás-partícula e o modelo concentrado para a secagem do bagaço de cana-de-açúcar, assumindo que a partícula era constituída apenas de água.

Neste sentido, o presente trabalho tem como proposta realizar um estudo teórico da secagem do bagaço de cana-de-açúcar usando um ciclone como secador, com um escoamento no sentido antihorário considerando a partícula como sendo uma mistura binária bagaço de cana-de-açúcar e água (sólido-líquido).

\section{MATERIAIS E MÉTODOS}

O fenômeno estudado no presente trabalho corresponde à secagem do bagaço de cana-de-açúcar em um ciclone, onde o ar aquecido passa por um alimentador no qual é introduzido o bagaço de canade-açúcar para, então, a mistura ar quente mais bagaço de cana-de-açúcar alimentar o ciclone secador através de um duto retangular (Figura 1a). A malha numérica constituída por 325.200 elementos hexaédricos (Figura 1b) gerada utilizando o ICEM CFD.
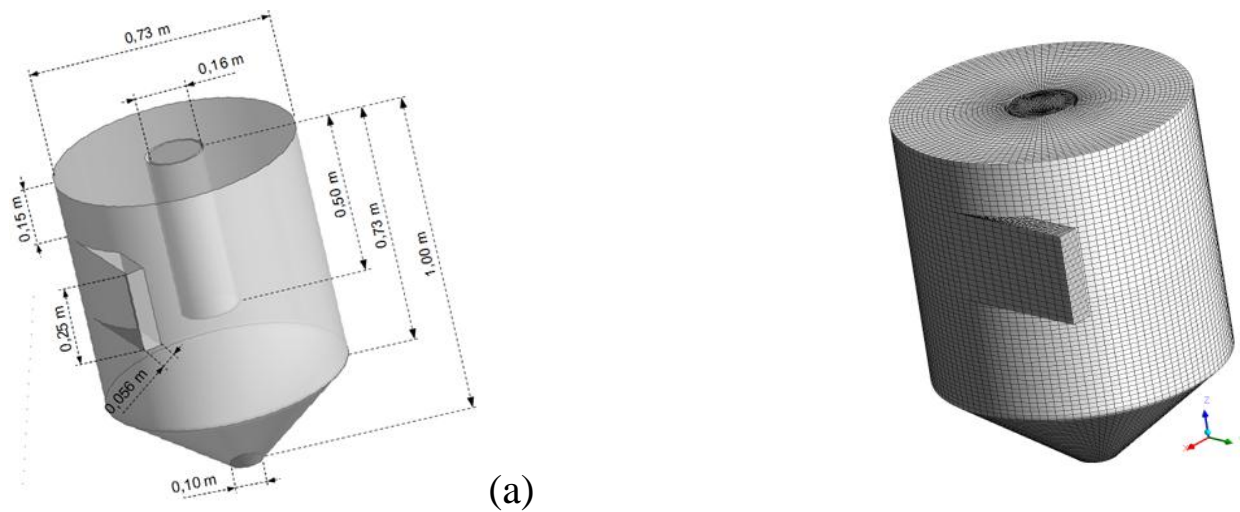

(a)

Figura 1 - (a) Modelo geométrico do ciclone baseado no trabalho de Corrêa (2003), (b) Representação da malha numérica.

\subsection{Modelo Matemático}


O modelo matemático usado para descrever o escoamento de partículas corresponde ao modelo multifásico Euleriano-Lagrangeano. Foram levados em considerações os seguintes pontos: regime permanente; não há reação química; foi considerado o efeito da gravidade; foi adotado o modelo de partículas; as propriedades físico-químicas são constantes; e a corrente gasosa (ar e vapor de água).

Equação da fase gasosa: Com estas considerações as equações de conservação de massa e momento linear (Equação 1 e 2) onde $\rho$ é a densidade da fase contínua e $\vec{u}$ é o vetor velocidade.

$$
\nabla \cdot(\rho \vec{u})=0
$$

Equação de momento linear:

$$
\nabla \cdot(\rho \vec{u} \vec{u})=-\nabla_{P}+\nabla \cdot \mu_{e f}\left(\nabla \vec{u}+(\nabla \vec{u})^{T}\right)+S_{M}
$$

onde, $S_{M}$ é o termo fonte, forças de corpo e de rotação, $\mu_{e f}$ é a viscosidade efetiva. Adotou-se o modelo padrão das tensões de Reynolds (LRR-IP) disponibilizado no aplicativo computacional ANSYS CFX.

Equação da Energia:

$$
\nabla \cdot\left(\rho \vec{u} h_{t o t}\right)=\nabla \cdot(\lambda T)+\nabla \cdot(\vec{u} \cdot \tau)
$$

onde, $\lambda$ é a condutividade térmica e $h_{\text {tot }}$ corresponde a entalpia total e o termo $\nabla .(\vec{u} . \tau)$ representa o trabalho devido às tensões viscosas (trabalho viscoso).

Equação de transferência de massa:

$$
\nabla \cdot\left[\rho \vec{u} Y_{A}-\rho D_{A}\left(\nabla Y_{A}\right)\right]=0
$$

onde, $Y_{A}$ corresponde a fração mássica do componente A; $D_{A}$ é a difusividade cinemática.

Equações de transporte de partículas: As partículas foram consideradas como sendo uma mistura binária (bagaço de cana-de-açúcar e água). A equação que representa a equivalência ou interação entre a fase gasosa e a fase particulada é dada pela 2a lei de Newton. A transferência de calor entre a partícula-ar de secagem é expressa empregando:

$$
\mathrm{Q}_{\mathrm{c}}=\pi \mathrm{d}_{\mathrm{p}} \lambda \mathrm{Nu}\left(\mathrm{T}_{\mathrm{G}}-\mathrm{T}_{\mathrm{p}}\right)
$$

onde, $\mathrm{T}_{\mathrm{p}}$ é a temperatura da partícula; $\lambda$ a condutividade térmica do fluido; $\mathrm{T}_{\mathrm{G}}$ é a temperatura do gás e $\mathrm{Nu}$ é o número de Nusselt definido como função dos números de Prandtl, Pr, e do número de Reynolds da partícula, $\operatorname{Re}_{p}$, dado pela correlação de Ranz e Marshall, citado no manual do CFX (2000) válida para $5 \times 10^{5} \leq \operatorname{Re}_{p} \leq 5 \times 10^{7}$ e $0,5 \leq P_{r} \leq 2000$.

Transferência de calor latente associado à transferência de massa : A energia consumida para evaporar a água na superfície da partícula, $Q_{m}$, é definida como sendo a somatória da energia de cada partícula que entra no ciclone dada por: 


$$
Q_{m}=\sum \frac{d m_{p}}{d t} h_{f g}
$$

o calor latente de vaporização, $h_{f g}$, em função da temperatura. A transferência de massa entre a fase particulada e a fase gasosa é determinada pela seguinte equação:

$$
\frac{d m_{p}}{d t}=\pi d_{p} \rho D S h \frac{P M_{v}}{P M_{g}} \log \left(\frac{1-X e}{1-X_{g}}\right)
$$

onde, $D$ é a difusividade dinâmica; $P M_{v}$ massa molar do vapor de água $(18 \mathrm{kj} / \mathrm{kmol}) ; P M_{g}$ a massa molar $\mathrm{r}$ do ar $(28,96 \mathrm{kj} / \mathrm{kmol}) ; X_{e}$ a fração molar de equilíbrio; $X_{g}$ a fração molar do gás e $S h$ é o número de Sherwood, com $K_{m}$ o coeficiente de transferência de massa; $D_{a g}$ é a difusividade do vapor de água no ar $L_{c}$ é o comprimento característico (V/A). O número de Sherwood foi determinado usando a correlação empírica $2+0,6 \operatorname{Re}_{p}{ }^{1 / 2} \mathrm{Sc}^{1 / 3}$ válida para $2 \leq \operatorname{Re}_{p} \leq 200$, $\mathrm{Sc}$ é o número de Schmidt, A pressão de vapor é determinada com auxílio da equação de Antoine, onde $\mathrm{A}, \mathrm{B}, \mathrm{C}$ são constantes e iguais a 18,$30 ; 3816,44$ e 46,13 respectivamente.

\section{Condições de contorno para a fase gasosa}

(a) Paredes do ciclone: Adotou-se a condição de não deslizamento para as componentes de velocidade e condição de fluxo de calor adiabático.

(b) Seção de entrada do duto de alimentação: Foi definido um perfil de velocidade da fase gasosa na seção transversal de entrada no duto de alimentação para a componente de velocidade na direção y, $u y=a+b z+c z^{2}+d z^{3}+e z^{4}+f z^{5}$ as outras componentes de velocidades nulas $u x=u z=0$, onde a, b, c, d, e, f são constantes e iguais a $(0,1458,-31327,264715,-1020055,1476460)$ respectivamente, obtidos a partir de um ajuste dos dados experimentais disponibilizados por Corrêa (2003).

(c) Saídas do ciclone: Adotou-se a condição de pressão prescrita e igual à pressão atmosférica e condição de Newmann para a temperatura, isto é, a variação de temperatura na direção normal ao contorno é nula.

Condições iniciais e de contorno para a fase sólida: Definiu-se inicialmente a fração de partículas, $\mathrm{f}_{\mathrm{p}}$, diâmetro das partículas, $\mathrm{d}_{\mathrm{p}}$, temperatura das partículas, $\mathrm{T}_{\mathrm{p}}$, e teor de umidade em base úmida, $\mathrm{M}_{\mathrm{bui}}$, onde seus valores são apresentados na Tabela 1. Na Tabela 2, constam os dados físico-químicos do processo de secagem.

Tabela 1 - Dados iniciais das partículas e ar de secagem

\begin{tabular}{|c|c|c|c|c|c|}
\hline Partícula & $\mathrm{f}_{\mathrm{pi}}(\%)$ & $\mathrm{d}_{\mathrm{pi}}(\mathrm{mm})$ & $\mathrm{T}_{\mathrm{pi}}(\mathrm{K})$ & $\mathrm{T}_{\mathrm{ar}}(\mathrm{K})$ & $\mathrm{M}_{\text {bui }}(\%)$ \\
\hline 1 & 1,4 & 0,42 & 298,0 & 489,0 & 76,19 \\
\hline 2 & 87,2 & 0,84 & 298,0 & 489,0 & 76,19 \\
\hline 3 & 2,2 & 2,75 & 298,0 & 489,0 & 76,19 \\
\hline 4 & 9,2 & 6,35 & 298,0 & 489,0 & 76,19 \\
\hline
\end{tabular}


Tabela 2 - Dados físico-químicos do processo de secagem.

\begin{tabular}{|l|c|c|}
\hline \multicolumn{1}{|c|}{ Propriedades físicas e químicas } & $\begin{array}{c}\text { Fluido: } \mathrm{ar}, \mathrm{M}_{\mathrm{bs}}=2,2 \\
\mathrm{~kg} / \mathrm{kg}) ; \mathrm{T}=489 \mathrm{k} .\end{array}$ & $\begin{array}{c}\text { Partícula (bagaço de } \\
\text { cana-de-açúcar) }\end{array}$ \\
\hline Densidade $\left(\mathrm{kg} / \mathrm{m}^{3}\right)$ & 0,7123 & 685,2 \\
\hline $\begin{array}{l}\text { Densidade da mistura (ar mais } \\
\text { bagaço de cana-de-açúcar) }\left(\mathrm{kg} / \mathrm{m}^{3}\right)\end{array}$ & - & 878,4 \\
\hline Condutividade térmica $(\mathrm{W} / \mathrm{m} . \mathrm{K})$ & $3,7673 \times 10^{-2}$ & 0,215 \\
\hline Calor latente de vaporização $(\mathrm{J} / \mathrm{kg})$ & & $2,251 \times 10^{6}$ \\
\hline Calor específico $(\mathrm{J} / \mathrm{kg} . \mathrm{K})$ & 1045,362 & 4180,0 \\
\hline Difusividade $\left(\mathrm{m}^{2} / \mathrm{s}\right)$ & $5,7960 \times 10^{-5}$ & - \\
\hline Temperatura de bulbo úmido $(\mathrm{K})$ & 324,81 & - \\
\hline Umidade relativa do ar $(\%)$ & 3,41 & - \\
\hline
\end{tabular}

\section{RESULTADOS E DISCUSSÕES}

Na Figura 3 está representado o perfil de velocidade da mistura (ar mais partículas) na entrada do ciclone na direção y em função da posição longitudinal z. Observa-se nesta figura que a Equação proposta se ajusta adequadamente aos dados obtidos por Corrêa (2003).

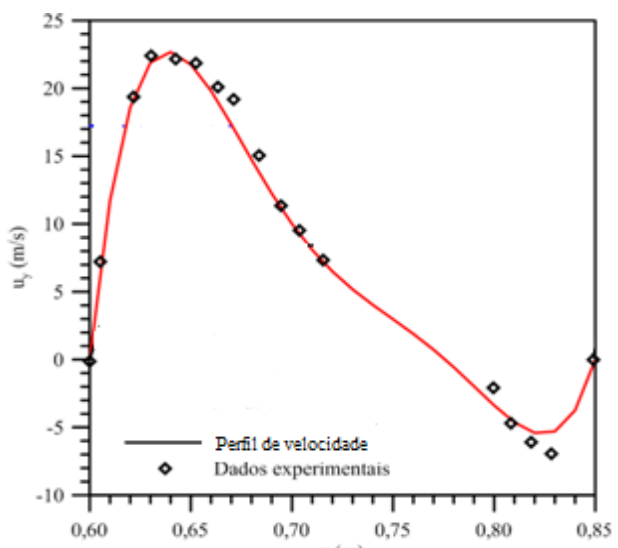

Figura 3 - Perfil de velocidade da mistura ar mais bagaço de cana-de-açúcar em função da posiçãı na seção de entrada do duto de alimentação do ciclone secador com base nos dados experimentais de Corrêa (2003)

Visando avaliar o efeito da posição do duto de alimentação sobre os perfis de velocidade, com fluxo (ar mais partículas) no sentido anti-horário estão representados nas Figuras 4 e 5 os perfis de velocidade axial e tangencial, dispostos dos lados direito e esquerdo no ciclone, em função da posição radial (posição y) usando uma malha, com a mesma distribuição e número de elementos utilizados na malha com o duto de alimentação posicionada no sentido horário. 


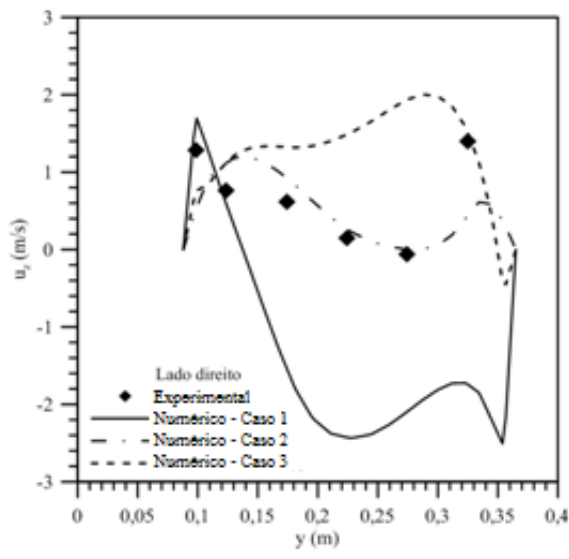

(a)

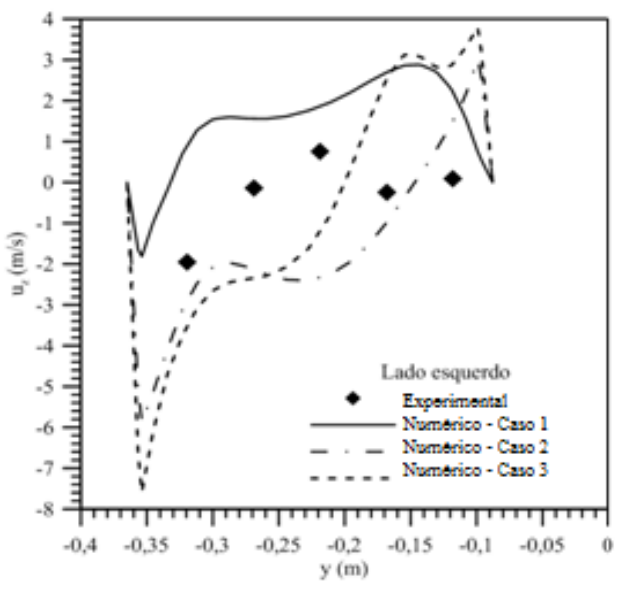

Figura 4 - Perfis de velocidade axial numérico e experimental para os casos 1,2 e $3\left(v_{p}=16,0\right.$; 18,0 e $20,0 \mathrm{~m} / \mathrm{s}$, respectivamente e $v_{\text {gás }}=20,0 \mathrm{~m} / \mathrm{s}$ ) com entrada no sentido anti-horário (a) do lado direito, (b) do lado esquerdo.
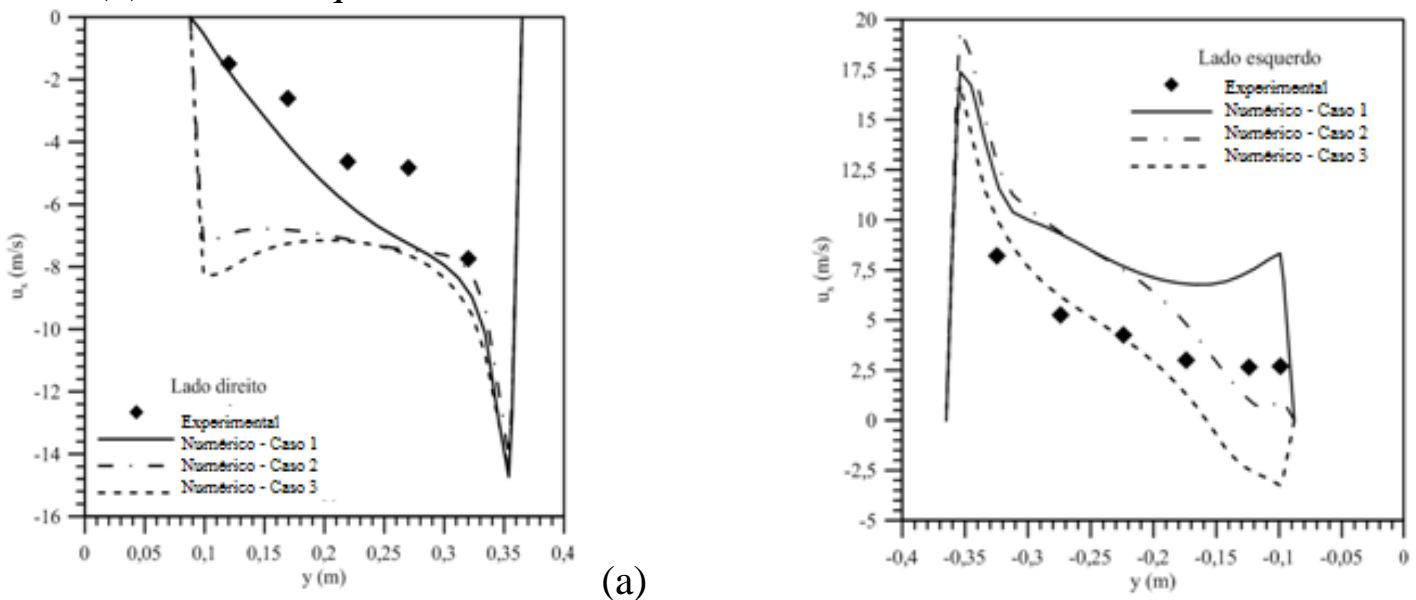

Figura 5 - Perfis de velocidade tangencial numérico e experimental para os casos 1,2 e 3 $\left(\mathrm{v}_{\mathrm{p}}=16,0 ; 18,0\right.$ e $20,0 \mathrm{~m} / \mathrm{s}$, respectivamente e $\left.\mathrm{v}_{\text {gás }}=20,0 \mathrm{~m} / \mathrm{s}\right)$ com entrada no sentido anti-horário. (a) do lado direito do ciclone, (b) do lado esquerdo do ciclone.

Os resultados apresentados nas Figuras 4 e 5 ilustram um comportamento similar aos apresentados no caso em que o duto de alimentação do ciclone está posicionado no sentido horário (Souza, 2012), para os perfis de velocidade axial, tanto do lado direito como do lado esquerdo. Para os perfis de velocidade tangencial, percebe-se um comportamento simétrico. Observam-se igualmente discrepâncias semelhantes ao se comparar com os dados experimentais disponibilizados por Corrêa (2003. Estes resultados põem igualmente em evidência a influência da velocidade das partículas sobre o comportamento da corrente gasosa, explicada pelas mudanças de comportamento nos perfis de velocidade axial e tangencial. Observa-se, portanto, que as componentes de velocidade axial e tangencial para o caso (velocidade das partículas de $16 \mathrm{~m} / \mathrm{s}$ ) apresentam uma discrepância bastante significativa das observadas para os casos 2 e 3 (velocidades das partículas de $18 \mathrm{~m} / \mathrm{s}$ e $20 \mathrm{~m} / \mathrm{s}$, respectivamente). 


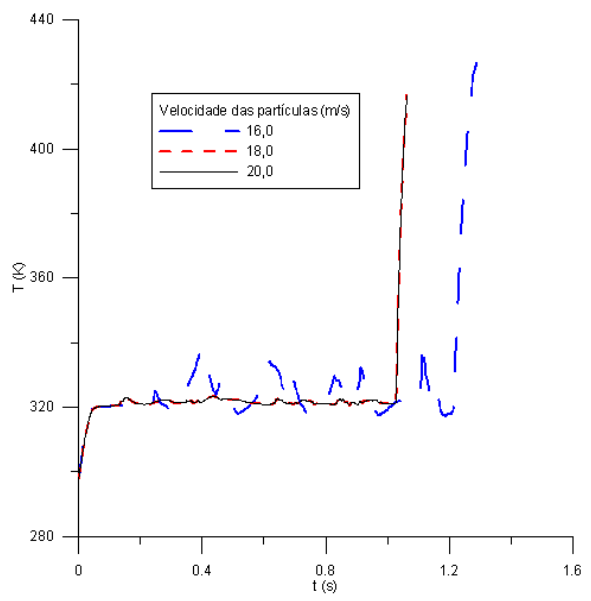

Figura 6 - Influência da velocidade de entrada da partícula no ciclone com sentido anti-horário so a temperatura.
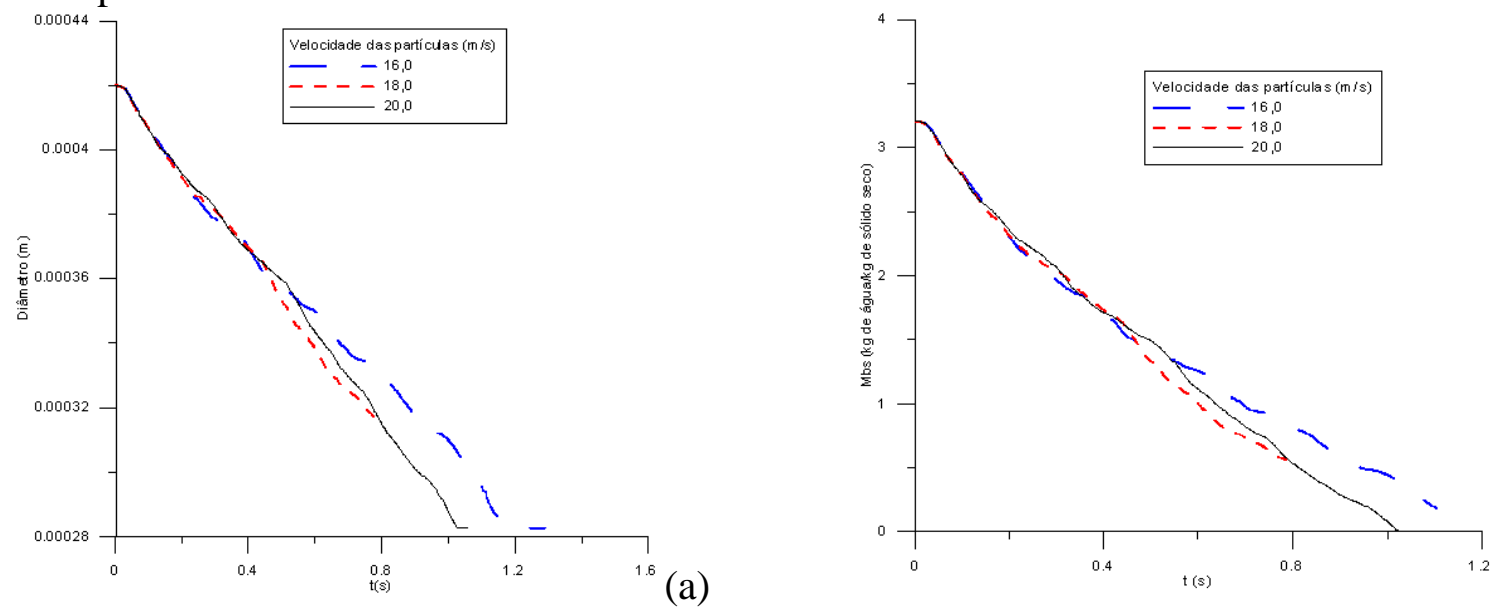

Figura 7 - Influência da velocidade de entrada da partícula no ciclone com sentido anti-horário sobre: (a) o diâmetro das partículas (b) o teor de umidade b.s.

Nas Figuras 6 e 7 estão representadas, respectivamente, a temperatura, diâmetro e teor de umidade em base seca das partículas de $0,42 \mathrm{~mm}$ em função do tempo de secagem no interior do ciclone com duto de entrada no sentido anti-horário, para as três velocidades de entrada das partículas no ciclone $(16 ; 18$ e $20 \mathrm{~m} / \mathrm{s})$ e mantendo-se a do ar de secagem igual a $20 \mathrm{~m} / \mathrm{s}$. Percebe-se nestas figuras que após $0,3 \mathrm{~s}$ um aumento repentino da temperatura das partículas para, em seguida, se manterem com um valor praticamente constante até sua saída do ciclone. Nota-se também que, próximo a um segundo, há um aumento brusco da temperatura, de 320 a $450 \mathrm{~K}$, até a sua saída do ciclone. $\mathrm{O}$ fato do diâmetro das partículas de $0,42 \mathrm{~mm}$ se manterem constante e igual a $0,282 \mathrm{~mm}$ (Figura 6) para tempos superiores a $0,9 \mathrm{~s}$ pode ser explicado pela ausência de água nas partículas possibilitando, assim, seu brusco aquecimento. Embora o modelo matemático proposto não contemple os fenômenos de combustão, degradação, tensões e choque entre partículas, pode-se conectar que esta brusca elevação da temperatura pode levar a queima das partículas do bagaço de cana-de-açúcar, a 


\section{9 a 22 de outubro de 2014 \\ Florianópolis/SC}

degradação ou ainda estar sujeita a tensões que podem comprometer a estrutura das partículas, o que não foi possível observar nos resultados numéricos em virtude do modelo não contemplar estes fenômenos.

Observa-se na Figura $7 \mathrm{~b}$ que o par de velocidades $20 \mathrm{~m} / \mathrm{s}$ a corrente de ar de secagem e $20 \mathrm{~m} / \mathrm{s}$ das partículas, é a situação mais adequada no processo de secagem, tendo em vista que as partículas de 0,42 mm saem com um teor de umidade em base seca nula. Destaca-se ainda nas Figuras 6 e 7 que o comportamento das curvas da temperatura, diâmetro e teor de umidade em base seca em função do tempo não são praticamente afetados pelas velocidades de entrada das partículas no duto de alimentação do ciclone.

\section{CONCLUSÕES}

Constatou-se que os perfis de temperatura, diâmetro e teor de umidade em base seca em função do tempo de permanência praticamente não são afetados pelas velocidades de entrada das partículas no duto de alimentação do ciclone, para partículas com diâmetro inicial de $0,42 \mathrm{~mm}$. O par de velocidades gás-partícula iguais $20 \mathrm{~m} / \mathrm{s}$ indicam ser a mais adequada no processo de secagem do bagaço de cana-de-açúcar nas condições avaliadas. O ciclone utilizado mostrou-se eficiente no processo de secagem do bagaço de cana-de-açúcar podendo ser explorado nas indústrias para melhorar a eficiência energética de caldeiras que possuem o bagaço de cana-de-açúcar como combustível.

\section{REFERÊNCIAS}

ARNAO, J. H.; OLIVEIRA, F. M.; CORRÊA, J. L. G.; SILVA, M. A.; NEBRA, S. A., "Sugar cane bagasse drying - a review", Proccedings of the 14th International Drying Symposium, v. B, p. 990997, 2004.

CORRÊA, J. L. G., "Discussão de parâmetros de projeto de secadores ciclônicos”, Tese de Doutorado, Faculdade de Engenharia Mecânica, UNICAMP, Campinas, 2003.

CORRÊA, J. L.G.; GRAMINHO, D.R.; SILVA, M. A.; NEBRA, S. A., "The cyclone dryer - a numerical and experimental analysis of the influence of geometry on average particle residence time", Brazilian Journal of Chemical Engineering, v. 21, no1, 2004.

FARIAS, F. P. M., "Estudo teórico da termofluidodinâmica em secadores ciclônicos", Tese de Doutorado, Engenharia de Processos, Universidade Federal de Campina Grande, Campina Grande, 2006.

MARIANI, V. C.; LIMA, A. G. B., COELHO, L. S., "Apparent thermal diffusivity estimation of the banana during drying using inverse method", Journal of Food Engineering, v. 85, p. 569-579, 2008.

SILVA, W. P.; PRECKER, J. W.; LIMA, A. G. B.; "Drying kinetics of lima beam (phaseolus lunatus) experimental determination and prediction by diffusion models", International Journal of Food Engineeri ng, v.5, n.3, p. 1-21, 2009.

SOUZA, J. A. R., "Secagem de sólidos via ciclones: modelagem e simulação", Tese de Doutorado, Engenharia de Processos, Universidade Federal de Campina Grande, Campina Grande, 2012. 